\title{
Baseline caries prevalence was the most accurate single predictor of caries risk in all age groups
}

\author{
Abstracted from \\ Mejàre I, Axelsson S, Dahlén G, Espelid I, Norlund A, Tranæeus S, Twetman S. \\ Caries risk assessment. A systematic review. \\ Acta Odontol Scand 2013 Sep 2. [Epub ahead of print] PubMed PMID: 23998481. \\ Address for correspondence: Ingegerd Mejàre, Swedish Council on Health Technology Assessment, \\ PO Box 3657, S-103 59 Stockholm, Sweden. E-mail: mejare@sbu.se
}

\section{Question: How accurate are risk assessments in predicting caries?}

Data sources The Cochrane CENTRAL and Medline databases and reference lists of identified were searched.

Study selection Prospective longitudinal cohorts or randomised controlled trials were included.

Data extraction and synthesis Study assessment and data extraction were carried out independently by at least two reviewers. The quality of studies was assessed using the QUADAS and AMSTAR tools. The overall quality of evidence was assessed using the GRADE approach.

Results 90 studies were included, seven were of high quality, 35 of moderate quality and the rest poor. The accuracy of multivariate models was higher for pre-school children than for schoolchildren/ adolescents. As the models had rarely been tested in independent populations their accuracy is uncertain. The single predictor baseline caries experience had moderate/good accuracy in pre-school children and limited accuracy in schoolchildren/adolescents. In general, the quality of evidence was limited.

Conclusions Multivariate models and baseline caries prevalence performed better in pre-school children than in school-children/ adolescents. Baseline caries prevalence was the most accurate single predictor in all age groups. The heterogeneity of populations, models, outcome criteria, measures and reporting hampered the synthesis of results. There is a great need to standardise study design, outcome measures and reporting of data in studies on caries risk assessment. The accuracy of prediction models should be validated in at least one independent population.

\section{Commentary}

This systematic review analysed current evidence for different caries risk assessment models and criteria. The included studies showed a large heterogeneity in design, outcomes, methods and results. Thus, the authors comprehensively presented available data, but were unable to draw a definite conclusion or to calculate synthetic effect estimates.

The topic - caries risk assessment - is one of utmost importance in times of attempted individualised dentistry and growing economic constraints, and is addressed at both dental practitioners and the scientific community. The authors followed established methodologies including an extensive search and selection process, risk of bias assessment and evidence grading. The compiled evidence emphasises one major problem in the field (one the authors state as well): the heterogeneity of scales, cut-offs, risk parameters, follow-up times and results does not allow performing a meta-analysis or drawing definite conclusions.

There are some key points to be highlighted:

1. Easy-to-obtain risk indicators like baseline caries experience and, to a lesser degree, socio-economic or socio-demographic status are useful to assess caries risk.

2. A detailed evaluation of proximal factors (nutritional habits, oral hygiene) seems to have only limited value for caries risk assessment, and elaborate tests involving bacterial counts or salivary analysis have only poor accuracy and, most likely, limited reliability.

3. Risk assessment is less accurate for adolescents than for young children, and it is even more uncertain how indicators like caries experience might predict root caries incidence or, generally, caries incidence in the elderly. Here, the assessment of other, less well investigated parameters (mobility and dexterity, psychosocial and general health) might have some value.

4. Lastly, it is worth highlighting that risk indicators, ie not strictly causally associated parameters, rather than risk factors are suitable for caries risk assessment: it is therefore not guaranteed and rather unlikely that - in the near future - a better understanding of the complex factors determining caries risk will automatically lead to better risk prediction.

In summary, there is no indication that assessing sophisticated (and expensive) proximal risk factors offers advantages over determining only the baseline caries experience. Adding many variables might improve the model, but is probably unrealistic in most clinical situations. The increasing complexity of risk profiles in later stages of life seems to increase the difficulty of caries assessment as well. Future studies should adhere to certain defined quality criteria and use calibrated, internationally accepted assessment criteria and cut-offs to eventually allow quantitative data synthesis. Reviews like the present one are of great value, since they offer a comprehensive overview of current evidence whilst highlighting the shortcomings of existing risk assessment approaches. Thus, they allow us to identify useful clinical tools and to point out how to improve the scientific basis in the field. In conclusion, the study can be recommended for both clinicians seeking to evaluate their risk assessment strategies and researchers striving to improve evidence-based decision-making in dentistry.

Falk Schwendicke

Department of Operative and Preventive Dentistry, Charité Universitätsmedizin Berlin, Berlin, Germany

Evidence-Based Dentistry (2013) 14, 102. doi:10.1038/sj.ebd.6400963 